\title{
Particle Correlations at RHIC - Scrutiny of a Puzzle
}

\author{
Sven Soff ${ }^{a}$, Steffen A. Bass ${ }^{b}$, David H. Hardtke ${ }^{a}$ and Sergey Y. Panitkin ${ }^{c}$ \\ ${ }^{a}$ Lawrence Berkeley National Laboratory, Nuclear Science Division MS70-319, \\ 1 Cyclotron Road, Berkeley, CA94720, USA \\ b Department of Physics, Duke University, Durham, NC27708, USA, and \\ RIKEN BNL Research Center, Brookhaven National Laboratory, Upton, NY11973, USA \\ ${ }^{c}$ Physics Department, Brookhaven National Laboratory, PO Box 5000, Upton, \\ NY11973, USA
}

We present calculations of two-pion and two-kaon correlation functions in relativistic heavy ion collisions from a relativistic transport model that includes explicitly a first-order phase transition from a thermalized quark-gluon plasma to a hadron gas. We compare the obtained correlation radii with recent data from RHIC. The predicted $R_{\text {side }}$ radii agree with data while the $R_{\text {out }}$ and $R_{\text {long }}$ radii are overestimated. We also address the impact of in-medium modifications, for example, a broadening of the $\rho$-meson, on the correlation radii. In particular, the longitudinal correlation radius $R_{\text {long }}$ is reduced, improving the comparison to data.

Two-particle correlations at small relative momenta have been predicted to be particularly sensitive to a phase transition from quark-gluon matter to hadronic matter [1]. For a first-order phase transition, larger hadronization times were expected to lead to considerably enhanced correlation radii, characterizing the space-time extension of the particle-emitting source, compared to, for example, a purely hadronic scenario. The radii should also depend on the critical temperature $T_{c}$, the latent heat, the initial specific entropy density or the initial thermalization time of the quark-gluon phase.

Here, we discuss relativistic transport calculations at RHIC energies that describe the initial dense stage by hydrodynamics [2] and the later more dilute stages by microscopic transport [3] of the particles. The two models are matched at the hadronization hypersurface [4]. In the hadronic phase the particles are allowed to rescatter and to excite resonances based on cross sections as measured in vacuum. For the initial dense (hydrodynamical) phase a bag model equation of state exhibiting a first-order phase transition is employed. Hence, a phase transition in local equilibrium that proceeds through the formation of a mixed phase, is considered. The details of this relativistic hybrid transport model can be found elsewhere [4].

We first briefly summarize the main conclusions obtained in previous work [5.6.7]. Then, we show in detail the results for pions when calculating explicitly the correlation functions from the source function of the transport model. These results are subsequently compared to calculations that take in-medium modifications into account and to experimental data 
for central $\mathrm{Au}+\mathrm{Au}$ collisions at $\sqrt{s}_{N N}=130 \mathrm{GeV}$ [8,9].

Model calculations have demonstrated that the freeze-out hypersurfaces of pions extend to rather large radii and times compared to the size of the mixed phase [7,5]. In this hadronic phase many soft collisions take place which hardly modify the single-particle spectra but have a strong impact on the correlation functions that measure the final freeze-out state. Studying this in detail leads to the following conclusions [7]: (i) The dissipative hadronic phase leads to a rather large duration of emission. (ii) The $R_{\text {out }} / R_{\text {side }}$ ratio, thought to be a characteristic measure of this emission duration, increases with transverse momentum. (iii) The specific dependencies of the interferometry radii on the QGP properties are rather weak due to the dominance of the hadronic phase. This even results in qualitative differences if calculations with and without this subsequent hadronic phase are compared (dependence on the critical temperature) [7].

The correlations of kaons provide a severe test of the pion data and have several advantages [6]. In particular, the kaon density is much lower than the pion density [10]. Hence, multiparticle correlations that might play a role for the pions are of minor importance for the kaons. Also, the contributions from long-lived resonances are under better control for kaons. The $R_{\text {out }} / R_{\text {side }}$ ratio for kaons is shown in Fig. 1 . The sensitivity to $T_{c}$ and to the specific entropy density (SPS vs. RHIC) is enhanced at larger transverse momenta $\left(K_{T} \sim 1 \mathrm{GeV} / \mathrm{c}\right)$. This enhanced sensitivity is also driven by a strong increase of the direct emission component (from the phase boundary) at high $K_{T}$ as shown in Fig. 2. More and more kaons (up to $\sim 30 \%$ ) escape the initial stages (unperturbed by the hadronic phase).

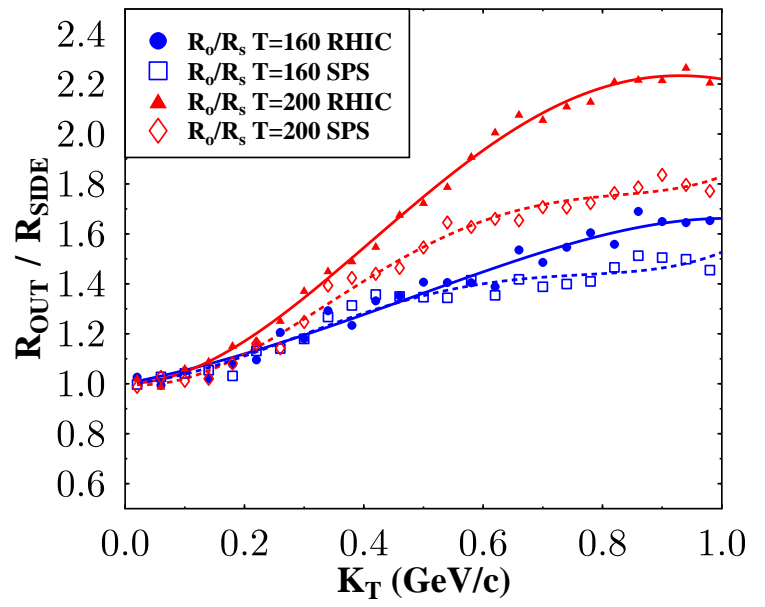

Figure 1. $R_{\text {out }} / R_{\text {side }}$ for kaons at RHIC (full symbols) and at SPS (open symbols), as a function of $K_{T}$ for critical temperatures $T_{c} \simeq 160 \mathrm{MeV}$ and $T_{c} \simeq 200 \mathrm{MeV}$, respectively.

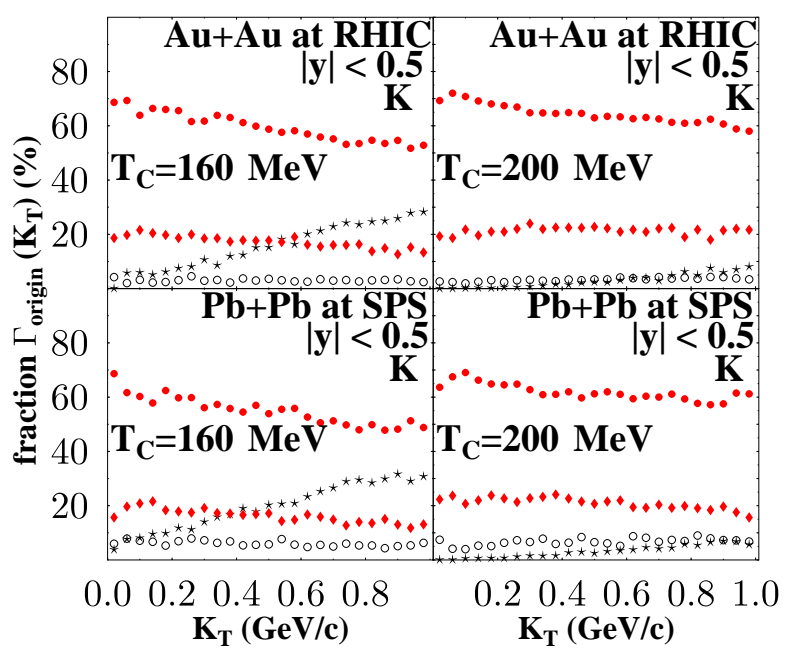

Figure 2. Fraction of kaons $\Gamma_{\text {origin }}$ that origin from a particular reaction channel prior to freeze-out. These are resonance decays (full circles), direct emission from the phase boundary (stars), elastic meson-meson (diamonds), or elastic meson-baryon (open circles) collisions. 


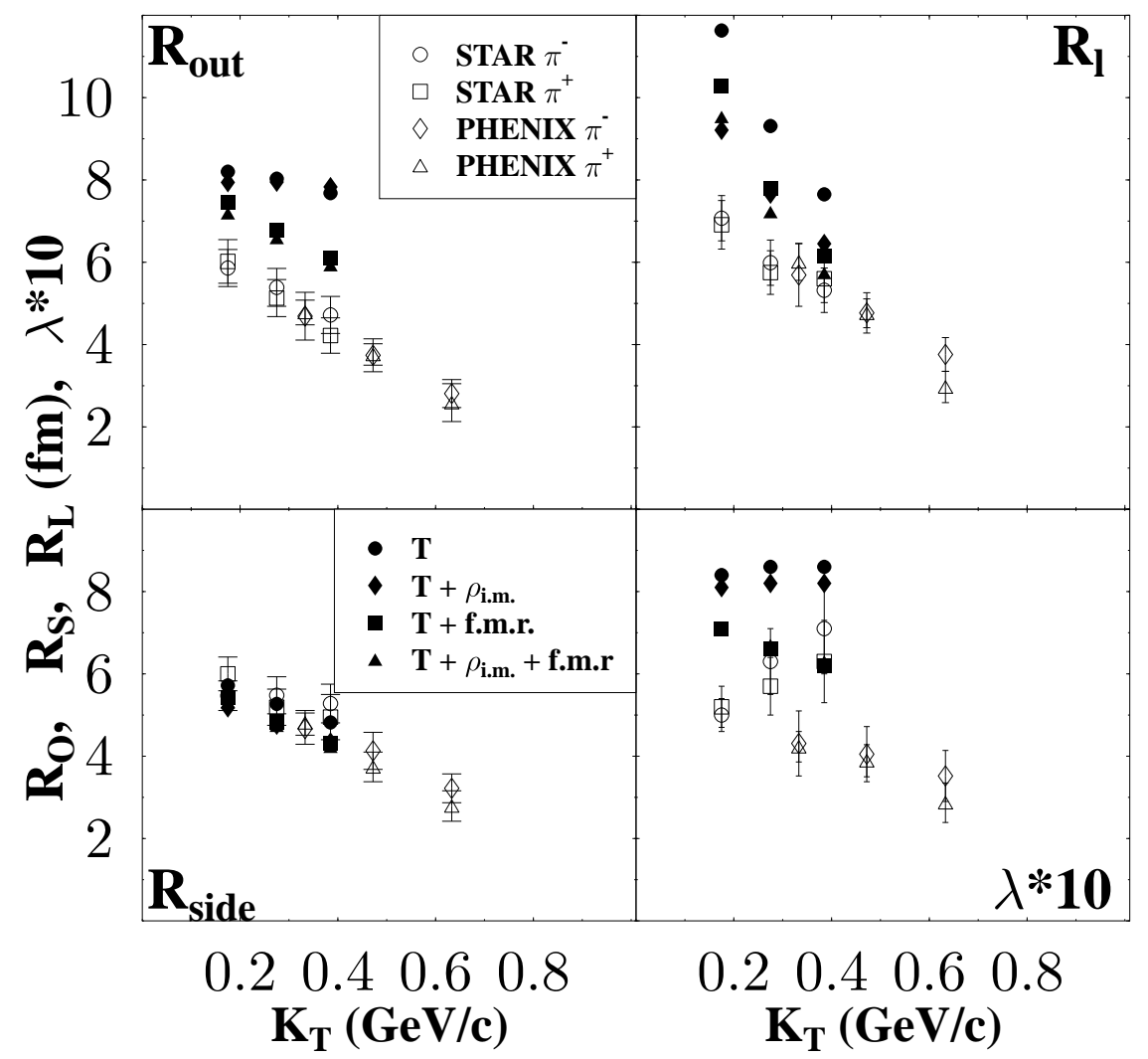

Figure 3. Pion correlation parameters $R_{\mathrm{o}}, R_{\mathrm{s}}, R_{\mathrm{l}}$, and $\lambda$ as obtained from the 'QGP+hadronic rescattering' transport model calculations + correlation after burner $(\mathrm{T})$ compared to experimental data from PHENIX and STAR. For theory (T), the effects of a finite momentum resolution (f.m.r.) and a broadened in-medium $\rho$ spectral function ( $\rho_{\text {i.m. }}$ ) are calculated. Note the strong reduction of $R_{l}$ when an in-medium $\rho$ is considered.

The pion correlation parameters $R_{\mathrm{o}}, R_{\mathrm{s}}, R_{1}$ and $\lambda$ are obtained from the explicit calculation of the correlation functions [11] in the respective transverse momentum bins and subsequent fitting of these correlation functions to a Gaussian form of the correlator $C_{2}=1+\lambda \exp \left(-R_{\mathrm{o}}^{2} q_{\mathrm{o}}^{2}-R_{\mathrm{s}}^{2} q_{\mathrm{s}}^{2}-R_{1}^{2} q_{\mathrm{l}}^{2}\right)$. The results of fitting the 3 -dimensional pion correlation functions (as obtained from the transport calculations (RHIC initial conditions, $\left.T_{c} \approx 160 \mathrm{MeV}\right)+$ correlation after burner (by Pratt) [11]) are shown, together with the experimental STAR and PHENIX data [8,9], in Figure 3. Even for a first-order phase transition scenario, the calculated interferometry radii $(\mathrm{T})$ are not unusually large. Finite momentum resolution (f.m.r.) reduces the true correlation radii and the $\lambda$ intercept parameter, in particular, at higher $K_{T}$ [6]. This is corrected for in the experimental analysis. While the $R_{\text {side }}$ radii show agreement the $R_{\text {out }}$ radii are too large compared to the data. However, these pion radii are considerably smaller than the corresponding radii obtained from the coordinate-space points and using expressions for the Gaussian radius parameters based on a saddle-point integration over the source function [5,6, :7, 12, Only the calculations as shown in Figure 3 which are obtained from the complete calculation and the performed fits should be compared to data. The $R_{\text {out }} / R_{\text {side }}$ ratio is also larger than unity for the fitted values and confirms the so-called HBT-puzzle, i.e., the RHIC data from STAR and PHENIX [8,9] indicate a decreasing ratio with $K_{T}$ (even below 1) 
and the calculations show only ratios larger 1 . We remark that at the CERN-SPS (see Refs. in [5]) the $R_{\text {out }} / R_{\text {side }}$ ratio seems to increase with $K_{T}$, being larger than 1, implying a real qualitative change of the reaction dynamics from SPS to RHIC energies. A discussion of theoretical assumptions and uncertainties, experimental corrections, and possible solutions of this problem (including strongly opaque sources or scenarios with strong supercooling leading to spinodal instabilities) is provided in [5].

In-medium modifications may alter the correlation radii due to their impact on the mean free path via modified cross sections at finite temparatures or densities $\left(T_{\text {f.o. }} \sim\right.$ $100-120 \mathrm{MeV})$. Increasing the width of the $\rho$ from the vacuum value of $\Gamma_{\rho}=150 \mathrm{MeV}$ to $\Gamma_{\rho}^{\text {i.m. }}=350 \mathrm{MeV}$ enhances the opacity of the system (more $\pi \pi \leftrightarrow \rho$ processes), with a particular reduction of the radius $R_{\text {long }}$, improving the comparison to data (Fig. 3).

We have demonstrated that the kaon interferometry measurements, in particular at high $K_{T}$, will provide an exellent probe of the space-time dynamics (close to the phase boundary). In addition they represent a severe test of the pion correlations and may help to better understand the HBT-puzzle [13], i.e., the difference in the $K_{T}$ dependence of the $R_{\text {out }} / R_{\text {side }}$ ratio between the model predictions and the experimental RHIC data. The differences are due to the $R_{\text {out }}$ radii (which are larger in the model calculations) while the $R_{\text {side }}$ radii seem to be described reasonably. An in-medium broadening of the $\rho$-meson reduces in particular $R_{\text {long }}$, improving the comparison to data.

Acknowledgments: $\quad$ SS is supported by the Alexander von Humboldt Foundation through a Feodor Lynen Fellowship and DOE Grant No. DE-AC03-76SF00098.

\section{REFERENCES}

1. S. Pratt, Phys. Rev. D 33, 1314 (1986); G. Bertsch, M. Gong, M. Tohyama, Phys. Rev. C 37, 1896 (1988); B. R. Schlei et al., Phys. Lett. B 293, 275 (1992); D. Rischke, M. Gyulassy, Nucl. Phys. A608, 479 (1996).

2. A. Dumitru and D. Rischke, Phys. Rev. C 59, 354 (1999).

3. S. A. Bass et al., Prog. Part. Nucl. Phys. 41, 255 (1998); M. Bleicher et al., J. Phys. G 25, 1859 (1999).

4. S. A. Bass, A. Dumitru, Phys. Rev. C 61, 064909 (2000).

5. S. Soff, Hirschegg 2002: Ultrarelativistic Heavy Ion Collisions, Hirschegg, Austria, 13-19 Jan 2002, (GSI, Darmstadt, 2002); hep-ph/0202240 and references therein.

6. S. Soff, S. A. Bass, D. Hardtke, S. Panitkin, Phys. Rev. Lett. 88, 072301 (2002) and J. Phys. G. 28, 1885 (2002).

7. S. Soff, S. A. Bass, A. Dumitru, Phys. Rev. Lett. 86, 3981 (2001).

8. STAR Collaboration, C. Adler et al., Phys. Rev. Lett. 87, 082301 (2001).

9. PHENIX Collaboration, K. Adcox et al., Phys. Rev. Lett. 88, 192302 (2002).

10. M. Murray, J. Phys. G 28, 2069 (2002).

11. S. Pratt et al., Phys. Rev. C 42, 2646 (1990); S. Pratt, Phys. Rev. Lett. 53, 1219 (1984); Phys. Rev. C 49, 2722 (1994); W. A. Zajc, Phys. Rev. D 35, 3396 (1987); S. Pratt et al., Nucl. Phys. A566, 103c (1994).

12. D. Hardtke, S. Voloshin, Phys. Rev. C 61, 024905 (2000).

13. D. Zschiesche et al., Phys. Rev. C 65, 064902 (2002); L. McLerran, S. Padula, nuclth/0205028; U. Heinz, P. Kolb, Nucl. Phys. A 702, 269 (2002); T. Hirano, K. Tsuda, nucl-th/0208068; D. Teaney, nucl-th/0209024; Z. Lin et al., nucl-th/0204054; T. Humanic, nucl-th/0205053; T. Csörgő, A. Ster, nucl-th/0207016. 